\title{
Direct Conversion Biogas to Multiwall Carbon Nanotubes and Syngas over Starch Derived Ni@C Nanoparticles.
}

\author{
Qiangu Yan ${ }^{1}$, Fei Yu ${ }^{1}$, I-Wei Chu², Amanda Lawrence ${ }^{2}$ \\ 1. Department of Agricultural and Biological Engineering, Mississippi State University, Mississippi \\ State, MS, USA \\ 2. Institute for Imaging and Analytical Technologies, Mississippi State University, Mississippi State, \\ MS, USA
}

Biogas is mainly composed of $\mathrm{CH}_{4}$ and $\mathrm{CO}_{2}$ [1]. The primary usage of biogas is as an internal combustion engine (ICE) fuel. Biogas is also studied to produce hydrogen as fuel cell feedstocks. An exciting alternative is to catalytic convert biogas to syngas which can be further upgraded to liquid fuels and chemicals through Fischer-Tropsch process. During $\mathrm{CH}_{4}-\mathrm{CO}_{2}$ dry reforming process, the most challenging problem is the catalyst deactivation by carbon deposition. This concern is even significant if using biogas directly, since the higher $\mathrm{CH}_{4} / \mathrm{CO}_{2}$ ratio (up to 1.5 1.6 for biogas) will end with higher and faster carbon formation over the catalyst that rapidly deactivates it. A new approach has been proposed to overcome this catalyst deactivation by converting the deposited carbon to a value-added product while avoiding the catalyst deactivation. The key issue of this procedure is to find a suitable catalyst to implement both the reforming reaction and the nano carbon structure formation since nano-carbons have been widely applied in material, electronic, energy storage, biomedical and environmental area. Nickelbased catalysts are widely used for both methane reforming and nano carbon formation processes. They promote the methane reforming reaction and effectively convert methane into highly valued products like carbon nanotubes, graphene and carbon nanofibers. This reactivity makes nickel-based catalysts an excellent choice to convert biogas into hydrogen-rich syngas and carbon nanostructures since biogas have relatively high $\mathrm{CH}_{4}: \mathrm{CO}_{2}$ ratios. The principal objective of this work was to produce and characterize multiwall carbon nanotubes (MWCNTs) and hydrogen-rich syngas from biogas over a highly efficient carbon-encapsulated metal nanoparticle catalyst.

Carbon-encapsulated nickel-core nanoparticles were synthesized hydrothermally by the reduction of $\mathrm{Ni}^{2+}$ from a starch solution. Six grams of $\mathrm{Ni}\left(\mathrm{NO}_{3}\right)_{2} \cdot 6 \mathrm{H}_{2} \mathrm{O}, 30 \mathrm{~g}$ starch and $250 \mathrm{ml}$ DI water were stirred for 30 min then transferred into a 500-mL Parr reactor; the reactor was heated up to $180{ }^{\circ} \mathrm{C}$ and kept for $8 \mathrm{~h}$. After reaction the resulting black product was collected and washed three times with DI water and ethanol. The final product was oven-dried at $110{ }^{\circ} \mathrm{C}$ overnight. The dried carbon-encapsulated nickel nanoparticles were calcined at $900{ }^{\circ} \mathrm{C}$ under a continuous nitrogen flow $(100 \mathrm{~mL} / \mathrm{min})$ for one hour. Catalytic conversion of biogas was carried out in a stainless steel tubular reactor $(0.0127 \mathrm{~m}$ O.D.) with $3 g$ of calcined nickel nanoparticles. The catalyst was first reduced at $400{ }^{\circ} \mathrm{C}$ under $\mathrm{H}_{2} / \mathrm{N}_{2}(1: 1)$ gas flow for $3 \mathrm{~h}$, then increasing the temperature to $850{ }^{\circ} \mathrm{C}$ feeding with $\mathrm{CH}_{4} / \mathrm{CO}_{2}$ gases $(100 \mathrm{~mL} / \mathrm{min})$ at a ratio of $3 / 2$ for one hour. The solid sample was collected and boiling in $0.1 \mathrm{M} \mathrm{HNO}_{3}$ solution for one hour. The prepared samples were characterized using microscopy to investigate the morphology, particle size and fine crystal structures. Samples for SEM were prepared by sprinkling the dried material on to double sided carbon tape affixed to AL stubs and coating with $10 \mathrm{~nm}$ PT (EMS $150 \mathrm{~T}$ coater). Samples were examined using a JEOL JSM 6500F microscope at $10 \mathrm{kv}$. For TEM the dried samples were mixed with $100 \% \mathrm{ETOH}$ and sonicated for $20 \mathrm{~min}$. A drop of the liquid was placed on a formvar/carbon 300 mesh copper grid for one hour then gently removed with filter paper. Grids were examined with a JEOL JSM 2100 microscope at $200 \mathrm{kv}$. X-ray powder diffraction (XRD) patterns of the synthesized samples were obtained using a Rigaku Ultima III X-ray Diffraction. 
SEM shows that the fresh nickel catalyst sample is spherical in shape with particle size between 1 and 2 $\mu \mathrm{m}$ (Fig.1a) whereas the micro-sphere calcined at $900{ }^{\circ} \mathrm{C}$ exhibits a porous structure (Fig.1b). After catalytic conversion biogas at $850^{\circ} \mathrm{C}$ for 1 hour Fig.1c shows a large amount of MWCNTs formed in the spheres. The purified sample (Fig.1d) clearly shows the MWCNTs with much higher purity with diameters ranging from 30 to $100 \mathrm{~nm}$. TEM shows MWCNT with a nickel particle (arrow) on its top (Fig. 2a). The diameter of the nickel particle was about $30 \mathrm{~nm}$. TEM image of MWCNTs indicates 50 to 100 carbon planes with the inter-layer spacing of $0.34 \mathrm{~nm}$ which corresponds to its graphite structure (Fig. 2b). MWCNTs formation is also confirmed by XRD, with the typical peaks for MWCNTs at $26.22^{\circ}$ and $53.97^{\circ} 2 \theta$ which corresponds to the (002) and (004) planes of graphite 2H. SEM, TEM and XRD results showed that biogas $\left(\mathrm{CH}_{4} / \mathrm{CO}_{2}\right.$ mixture) can be efficiently converted to MWCNTs and syngas over starch derived Ni@C nanoparticles.

\section{References:}

[1] S Rasi et al, Energy, 32 (8) (2007), p. 1375.

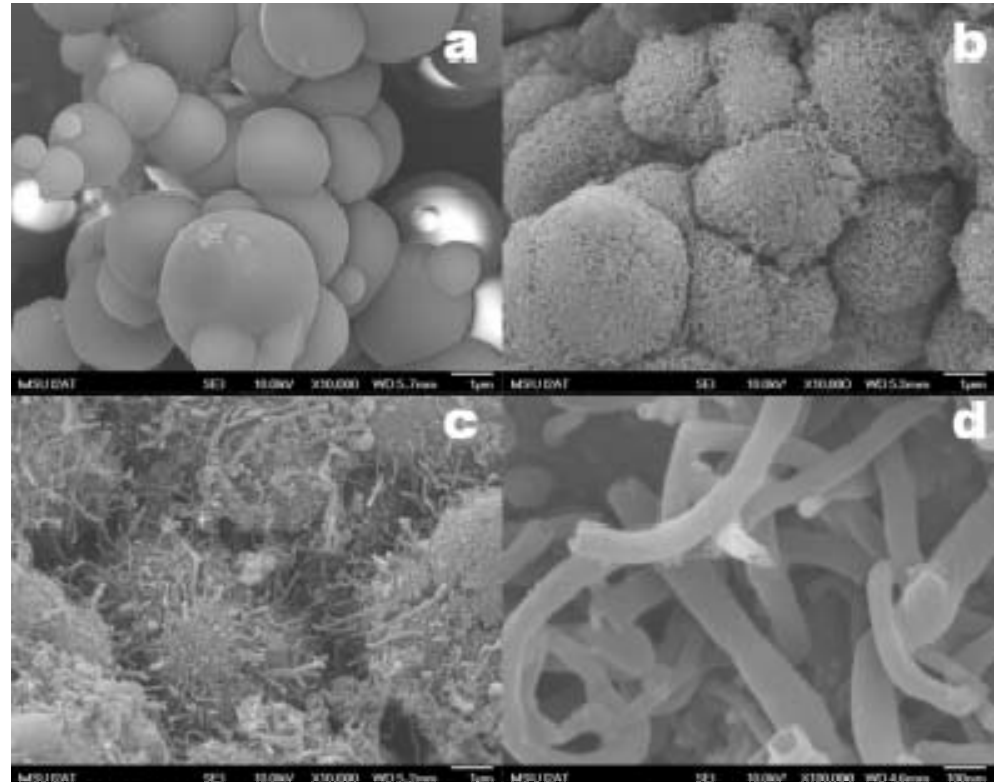

Figure 1. SEM images of the carbon-encapsulated nickel nanoparticles. a: the dried sample; b: the calcined sample; c: the catalytic conversion sample; d. purified sample.

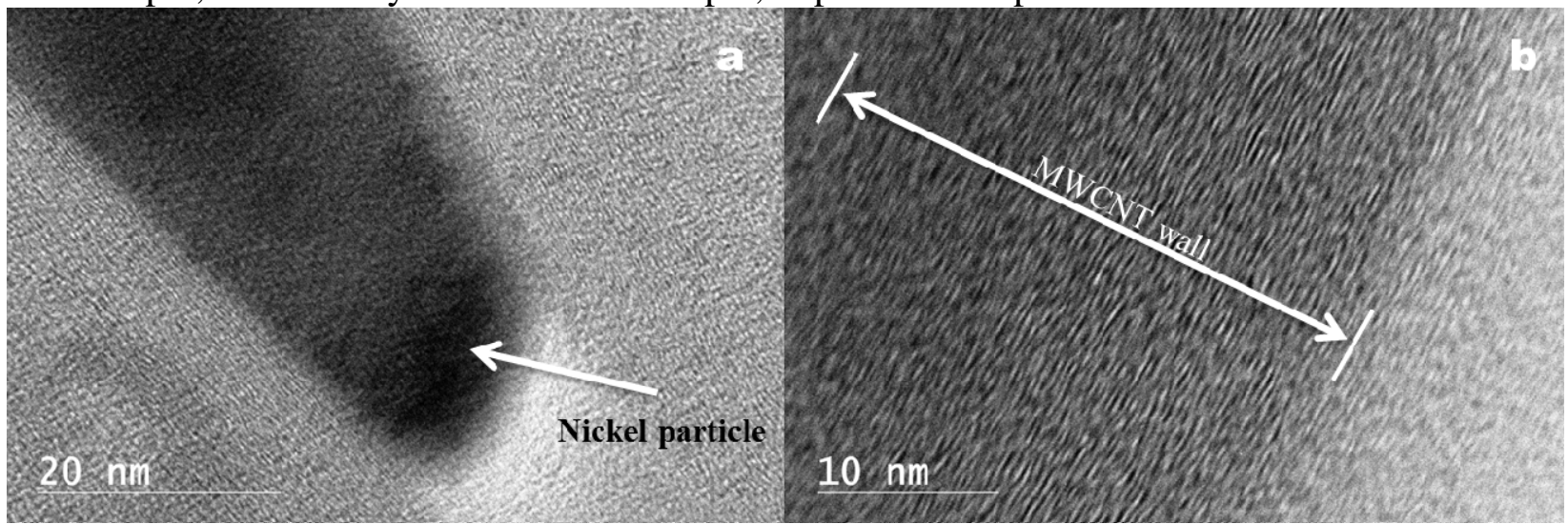

Figure 2. TEM images of MWCNTs formed during biogas conversion. a: MWCNTs with nickel nanoparticle on the top (arrow); b. the graphite plane structure of the MWCNT wall. 